\title{
PERIÓdICOS E RANKINGS DE PERIÓdICOS EM ADMINISTRAÇÃo
} JOURNALS AND JOURNAL RANKINGS IN MANAGEMENT

\author{
Recebido em 20.10.2014. Aprovado em 12.05.2015 \\ Avaliado pelo sistema double blind review \\ DOI: http://dx.doi.org/10.12712/rpca.v9i2.502
}

\section{Manoel Portugal Ferreira}

manuel.portugal.ferreira@gmail.com

Escola Superior de Tecnologia e Gestão - Instituto Politécnico de Leiria, PORTUGAL

Universidade Nove de Julho - PPGA, São Paulo/SP, BRASIL

\section{Resumo}

Publicar é essencial para os pesquisadores e as universidades, e publicar em periódicos internacionais com revisão pelos pares e ranqueados é, pelo menos, uma ambição crescente dos pesquisadores brasileiros em Administração. Os rankings de periódicos são relevantes para os pesquisadores, universidades, agências reguladoras e instituições financiadoras. Os rankings de periódicos são usados diretamente na avaliação da qualidade dos artigos científicos dos pesquisadores e, indiretamente, contribuem para a avaliação da atratividade de uma universidade, ou programa, para estudantes, professores, reguladores e financiadores. Apesar de amplamente usados e informalmente debatidos, poucos pesquisadores conhecem os rankings, suas implicações e o que realmente significam. Neste artigo discuto algumas facetas dos rankings de periódicos em Administração, incluindo a sua utilidade, apresento alguns rankings, e debato problemas no sistema. Se os rankings de periódicos cada vez mais orientam os esforços dos pesquisadores, é necessário um debate mais alargado que incida sobre seus sistemas, virtudes e disfunções. Este debate permitirá, por um lado, desmistificar e, por outro, esclarecer à comunidade e, especialmente, aos menos conhecedores da importância de avaliar os periódicos antes de submeter suas pesquisas.

Palavras-chave: Ranking de periódicos. Periódicos. Avaliação pelos pares. Qualis.

\begin{abstract}
Publishing is crucial for researchers and universities, and publishing in international peer reviewed journals is becoming, at least, a growing ambition of many management scholars. Journal rankings are relevant for faculty, universities, and financial and regulatory agencies. Journal rankings are used directly in assessing the quality of researchers' scientific output and, indirectly, they influence the attractiveness of universities, or departments, to new students, professors, regulators and financiers. Albeit widely used and informally debated, few are aware of the journal rankings, implications and what they really mean. In this paper, I discuss some of the facets involving the existence of journal rankings in management, including their usefulness, present some rankings and debate problems in the system. If journal rankings increasingly guide scholars' research efforts, a broader debate is warranted on the systems, virtues and dysfunctions. This debate will contribute, on one hand, to demystify, and on the other to elucidate the community, especially those less knowledgeable of the importance of assessing journals' quality prior to submitting their research.
\end{abstract}

Keywords: Journal rankings. Journals. Peer-review. Qualis. 


\section{Introdução}

Publicar ou perecer. Publicar é fundamental para a progressão na carreira acadêmica, para o status como membro da comunidade, para a satisfação pessoal e para benefícios complementares (HOJAT ET AL., 2003; ROND; MILLER, 2005). Mais do que publicar, crescentemente está se tornando importante conseguir publicar em periódicos nacionais e internacionais de alta reputação, com revisão pelos pares, e ranqueados (ou com fator de impacto). No entanto, não é fácil publicar (SERRA; FIATES; FERREIRA, 2008), nem é fácil publicar nos melhores periódicos internacionais (SAMKIN, 2011). Dada a proliferação de novos periódicos, também parece ser mais difícil identificar para quais periódicos submeter os artigos. Assim, entender para que serve os rankings de periódicos e o que estes rankings sinalizam é importante para os pesquisadores.

$\mathrm{Na}$ última década assistimos ao surgimento de vários rankings de periódicos (SHUGAN, 2003; GEARY ET AL., 2004; AZAR; BROCK, 2008; STEWARD; LEWIS, 2010; CURRIE; PANDHER, 2011; HARZING, 2013), por todo o mundo. Estes rankings são extensamente usados para avaliar diversas dimensões cruciais aos pesquisadores e às instituições (universidades, agências de fomento e reguladoras) (STAHL; LEAP; WEI, 1988; DE MEIS ET AL., 2003; TREVINO ET AL., 2010; SAMKIN, 2011). No Brasil a publicação e os rankings de periódicos também são relevantes (DE MEIS ET AL., 2003; CABRAL; LAZZARINI, 2011). Por exemplo, influenciam diretamente o conceito CAPES da universidade e a reputação científica dos pesquisadores. Atualmente, o sistema vigente avalia a qualidade dos artigos publicados como função da qualidade dos periódicos em que foram publicados (PFEFFER; FONG, 2004). Assim, os rankings - usados como indicadores de qualidade dos periódicos - influenciam aspectos como a obtenção de bolsas de produtividade e a concessão de apoios à pesquisa. Portanto, os rankings podem ser criticados, e sua importância e relevância debatidas (LEYDESDORFF, 2008; FARIA, 2011; BERTERO ET AL., 2013) - Clark e Wright (2007) referiram-se mesmo a "um sistema fora de controle" (tradução minha) -, mas não é possível ignorar que muito depende deles.

Neste artigo, por meio de um ensaio conceitual, visa-se lançar um debate mais alargado que será importante, pelo menos para a nova geração, de atuais mestrandos e doutorandos. Este debate ajuda a esclarecer aspectos como a importância de publicar nos melhores periódicos, inclusive internacionais, qual a utilidade e quais os rankings de periódicos, e o que significam indicadores como o fator de impacto. Outros aspectos relevantes incluem o entendimento das virtudes e disfunções potenciais do sistema atual (LEYDESDORFF, 2008; BERTERO ET AL., 2013; FREY, 2003; FARIA, 2011). Este artigo tem uma contribuição evidente para a comunidade e talvez mais especialmente para os estudantes de mestrado e doutorado stricto sensu e pesquisadores mais jovens. A realidade é que muitos destes (jovens) pesquisadores não conhecem os rankings e o que está por detrás deles. Uma segunda contribuição é para o debate sobre a utilização dos rankings, onde há um espectro de posições desde os que discordam da sua existência até aos ferozes defensores da sua utilização. Por fim, há uma contribuição para as instituições na medida em que, por um lado, as práticas institucionais são muito diferentes e, por outro, o foco exclusivo em periódicos internacionais com impacto, se adotado como a real métrica na avaliação da qualidade dos pesquisadores e programas, pode agravar as disparidades para além do desejável. O debate acadêmico é, assim, necessário.

Nas seções seguintes, debate-se, primeiro, a utilidade dos rankings. Depois, apresentam-se de forma sintética alguns rankings e inclui-se o Qualis Brasileiro e suas origens. $\mathrm{Na}$ terceira seção, apontam-se alguns cuidados a ter nos periódicos. Conclui-se com uma discussão que inclui sugestões de pesquisa futura.

\section{Porque temos Rankings e sua utilidade}

Estudos analisando, criticando ou propondo a utilização de rankings de periódicos vem tornandose mais comuns. Talvez esta atenção seja reflexo da maior preocupação com o impacto que têm sobre os pesquisadores e instituições (ROND; MILLER, 2005; BERTERO ET AL., 2013; DAVIS, 2014) em especial, a avaliação dos artigos do pesquisador inferida pela "qualidade" dos periódicos onde seus artigos foram publicados. Mas, também, pela forma como os rankings são construídos, o processo de avaliação dos periódicos e as suas disfunções (SHUGAN, 2007; TREVINO ET AL., 2010; LEWIN, 2014). Assim, nas diferentes disciplinas encontramos estudos que propõem rankings e avaliam 
os periódicos. Por exemplo, na disciplina de Negócios Internacionais (DUBOIS; REEB, 2000), Marketing (BAUMGARTNER; PIETERS, 2003; STEWARD; LEWIS, 2010), Estratégia (AZAR; BROCK, 2008), Turismo (PECHLANER ET AL., 2004) e Sistemas de Informação (PEFFERS; TANG, 2003).

Mas, qual a utilidade dos rankings? Os rankings servem múltiplas funções para os diversos agentes envolvidos: pesquisadores (e estudantes de mestrado e doutorado) universidades, agências de fomento e instituições reguladoras. Para todos estes agentes, os rankings servem como métrica que reduz a incerteza e proporciona critérios conhecidos (ainda que criticáveis e criticados) para a avaliação. A existência de rankings é justificada como forma expedita de avaliar (inferindo) a qualidade. Esta necessidade tornase especialmente importante quando pode haver alta subjetividade - que levaria diferentes pesquisadores a avaliar diferenciadamente os artigos e os periódicos ou quando é simplesmente impossível conhecer todos os periódicos.

Por exemplo, para os pesquisadores, os rankings são usados como métrica de reputação, reconhecimento pelos pares e progresso profissional, servindo para avaliar a qualidade das publicações nas decisões de contratação (TREVINO ET AL., 2010), para a premiação (bolsas de produtividade) e até para benefícios financeiros diretos (prêmios por artigo publicado que são ajustados à classificação do periódico). Os rankings sinalizam aos pesquisadores quais são os periódicos que a universidade (ou departamento) valoriza orientando os seus esforços de publicação. Os rankings permitem reduzir a incerteza dos pesquisadores e planejar os seus esforços de pesquisa - por exemplo, avaliando se determinado tipo de pesquisa poderá ser publicada nos periódicos usados como referência. Certamente há problemas neste sistema, por exemplo, quando um pesquisador não realiza pesquisa no mainstream (BERTERO ET AL., 2013) e que dificilmente será publicada nos periódicos de topo da área ou o pesquisador que tem um foco bem específico numa área que não é dominante no departamento.

Se a escolha do periódico pode ser difícil para pesquisadores experientes, então para estudantes de doutorado será quase dantesca. Os rankings nos oferecem uma indicação da qualidade relativa dos periódicos que funciona como uma heurística que ajuda na escolha. Simplificando: melhor classificação significa melhor periódico. Este pressuposto não tem sido livre das críticas de inúmeros pesquisadores (UNCLES, 2004; ROND; MILLER, 2005; SHUGAN, 2007). Duas disfunções podem emergir aqui. Uma é como distinguimos a qualidade dos periódicos. A segunda disfunção quando nos orientamos exclusivamente por critérios de rankings perdendo de vista a geração de conhecimento e a ciência. Ou seja, a publicação em periódicos de topo torna-se o fim em si mesmo.

Os rankings também são úteis para as instituições. No entanto, os rankings de periódicos têm atualmente um grande impacto institucional (CLARK; WRIGHT, 2007) sobre as universidades. Para as universidades, os rankings influenciam em sua reputação (BADENFULLER ET AL., 2000; SHUGAN, 2003; TREVIÑO ET AL., 2010) e em sua atratividade para recrutar professores e alunos. Isto porque cada vez mais os rankings são usados para avaliar a produção científica dos professores nos programas. Os rankings representam também um dos fatores usados mesmo nas populares listas do Financial Times. Assim, muitas universidades norte americanas, europeias e australianas já usam rankings nas decisões de contratação e progressão na carreira, ainda que com práticas distintas (ver HARZING, 2014). Ou seja, a avaliação dos pesquisadores é feita olhando para os rankings dos periódicos onde publicaram e não lendo (não avaliando) os artigos em si. Se as listas fossem idênticas seria inclusive possível comparar o desempenho científico de diferentes escolas ou departamentos.

De igual modo para outras agências, como as de fomento à pesquisa ou de regulação, a existência de rankings permite uma avaliação mais objetiva e mais rápida, já que dispensa a leitura e análise individualizada das publicações dos pesquisadores.

\section{Histórico dos ranqueamentos dos periódicos}

O histórico internacional da classificação (ou ranqueamento) de periódicos passou, num momento inicial, pela conceptualização do fator de impacto (RITZBERGER, 2008). A necessidade de uma forma de classificar periódicos surgiu dos bibliotecários americanos, que precisavam discernir quais eram os periódicos a adicionar aos seus acervos (ARCHAMBAULT; LARIVIERE, 2009). Os primeiros a estabelecer um ranking de periódicos foram Gross e Gross (1927), em um trabalho com o intuito de estabelecer uma lista como referência para 
bibliotecários. A primeira tentativa bem-sucedida de criar uma métrica para o impacto de artigos científicos na academia foi de Eugene Garfield (1955). Em essência, com o uso do número de citações (e número de citações dividido pelo número de artigos), Garfield (1955) pretendia oferecer à academia uma métrica que eliminasse incertezas sobre a qualidade dos artigos e periódicos a serem citados. Assim, uma das consequências previstas era que, com o conhecimento do impacto de um artigo ou periódico, os pesquisadores poderiam evitar material fraudulento ou obsoleto.

Em 1961, o Science Citation Index foi criado para mensurar as citações aos artigos publicados. Mais tarde este mesmo índice foi rearranjado e passou a ser utilizado pelo Journal Citation Index como métrica de avaliação da qualidade dos periódicos. Assim, em 1969 foi publicado o primeiro ranking de periódicos, ordenado pelo seu fator de impacto (GARFIELD, 2006). Gradualmente as métricas foram ampliadas, adicionando novas formas de análise, como seja a meia-vida das citações dos artigos. Atualmente, os periódicos são amplamente ranqueados baseando-se no Journal Citation Reports, que utiliza a mesma lógica do número de citações como numerador e do número de artigos como denominador (GARFIELD, 2006).

As formas de ranquear os periódicos, adotadas em diferentes países e instituições, podem utilizar de várias métricas que foram sendo desenvolvidas ao longo dos anos. Por exemplo, formas distintas de aferição de impacto. Encontramos, assim, atualmente, mensurações baseadas na porcentagem de artigos não citados (share of uncited papers), o índice $\mathrm{H}$ (que inicialmente foi concebido apenas para analisar o impacto de pesquisadores, mas, posteriormente, foi adaptado para medir o impacto de periódicos), o método BT (que leva em consideração as probabilidades de um periódico citar outro), e o método LP (que tem em consideração o peso de cada citação,em função do impacto do periódico onde foi citado (RITZBERGER, 2008). Porém, embora existam diferentes mensurações, internacionalmente o índice JCR é reconhecido como a principal métrica (ARCHAMBAULT; LARIVIERE, 2009) de avaliação de artigos e periódicos.

O Brasil não ficou imune a tendência internacional de utilizar medidas de avaliação da produção científica. No Brasil, o Qualis foi inicialmente estabelecido em 1998, com o intuito de aferir a qualidade das publicações em periódicos (FRIGERI; MONTEIRO,
2012). Nas três primeiras avaliações trienais, os rankings do Qualis tinham como objetivo primeiro demonstrar a qualidade e abrangência dos periódicos, dividindo-os em Internacionais, Nacionais e Locais, nos estratos A1, A2, A3, B1, B2, B3, C1, C2 e C3 (DINIZ, 2010; CAMPOS, 2010). Esta denominação utilizada no sistema Qualis que classifica em estratos A, B e C também foi utilizada para classificar os eventos científicos, mas o Qualis de eventos de extinguiu quando estes passaram a não ser reconhecidos como fonte de pontuação pela CAPES.

$A$ adoção de uma ordem mais linear dos estratos de periódicos - em A1, A2, B1, B2, B3, B4, B5 e C emergiu na avaliação trienal de 2007-2009 (DINIZ, 2010; CAMPOS, 2010) e permanece até o presente momento. $\mathrm{O}$ atual sistema de classificação passou a levar em conta fatores de organização do periódico, a indexação em bases de dados e o fator de impacto dos periódicos (MARTINS et al., 2014) ao invés dos critérios de qualidade e abrangência previamente utilizados (a Tabela 3 descreve os critérios em vigor no período 2010 a 2012). Os critérios de classificação, que diferenciam um periódico A1, por exemplo, de um B1 são definidos por cada área de estudo da CAPES, por uma comissão de pesquisadores de cada área (GOMES, 2010). As avaliações ocorrem periodicamente, e os critérios são revistos anualmente nos "documentos de área" publicados e disponibilizados publicamente para que todos os pesquisadores, mas, também, editores de periódicos, os possam conhecer e consultar.

\section{Evolução do número de periódicos}

No Brasil, o número de periódicos em Administração teve um forte crescimento na última década, e temos atualmente mais de 200 periódicos nacionais classificados em Administração no Qualis. A maioria destes periódicos existe apenas em formato online, o que oferece dois benefícios primordiais: os custos para a instituição são mais baixos (consideravelmente menores que os implicados na impressão e distribuição do formato físico em papel), e a muito maior facilidade de divulgação dos artigos. Os periódicos brasileiros, de maneira geral, não contém restrições de acesso. Este formato, que é usualmente designado por open access, ou acesso livre, tem sido promovido face aos altos custos que podem estar envolvidos na assinatura dos periódicos tornando-o incomportável para as instituições restringindo o acesso aos pesquisadores e ao público maior. 
Com o aumento do número de periódicos em Administração, ou que publicam artigos em Administração, é cada vez mais difícil para os autores escolher o periódico onde submeter os seus trabalhos de pesquisa. A multiplicação de periódicos também levanta outros problemas. Em especial os problemas de qualidade (muitos periódicos são francamente fracos) e de real respeito pelas normas aceitas pela comunidade para o trabalho científico - especialmente, a revisão pelos pares. Em outros casos, muitos dos periódicos têm fraco suporte institucional, são mal geridos e não garantem continuidade.

Embora não identifique uma lista completa com todos os periódicos existentes (ver uma discussão em Larsen e Von Ins, 2010), há algumas indicações que a publicação científica tem vindo a aumentar e, em algumas áreas, de forma muito substancial. Vale notar alguns indicadores. Segundo Björk et al. (2008) o número de periódicos com revisão pelos pares aproximava-se dos 24.000 em 2008. Em 2008 a Scopus incluía 15.800 periódicos, a SCIE 6.650 e a SSCI 1.950 periódicos (BJÖRK ET AL., 2008). Ware e Mabe (2008) reportam que em 2012 existiriam cerca de 28.100 periódicos acadêmicos com revisão pelos pares ativos e que, no conjunto, publicavam cerca de 1,8 ou 1,9 milhões de artigos anualmente.

A internet veio promover, quase que absurdamente, a criação de novos periódicos. Ao baixar as barreiras à entrada - e em especial os custos e a facilidade de divulgação - o número de periódicos multiplicou-se (e continua a aumentar) em formatos digitais online. No essencial são as baixas barreiras à entrada que as TIC proporcionam que facilitam este movimento ascendente, mas importa também entender o que estamos a tratar porque há realidades muito distintas entre os periódicos de acesso livre. Entre os novos entrantes há muitas iniciativas respeitáveis, mas, também, há muitos periódicos que requerem um pagamento para publicação que pode ser bastante substancial (em alguns casos 500 a 800 dólares americanos, uma notícia no New York Times de 2013 reporta que o custo pode ir até 2.700 dólares por artigo) e potencialmente com pagamento extra para figuras ou páginas adicionais). Jeffrey Beall, da Universidade do Colorado, construiu uma lista de periódicos que designa por "Potenciais, possíveis, ou prováveis periódicos predatórios de acesso livre" (tradução minha) - esta lista está disponível publicamente http://scholarlyoa.com/individualjournals/. É interessante observar que nesta lista os periódicos têm nomes pomposos como "American
Journal...", "European Journal..." e especialmente "International Journal...". O problema potencial não está na meritória disponibilização gratuita dos artigos que fundeou o movimento do acesso livre, mas sim no aproveitamento de algumas empresas e indivíduos para ganho pessoal.

Ou seja, em muitos casos, estes novos periódicos não "partilham o idealismo do movimento de acesso livre" (tradução minha) (DAVIS, 2014, p. 196). São periódicos pouco críveis. Na realidade, é provável que o leitor receba diariamente ofertas destes periódicos para publicar o seu artigo, para fazer parte do seu corpo editorial ou até mesmo receba convites para ser o editor. Parte da dificuldade então para os agentes - pesquisadores, universidades, instituições - é como distinguir qualidade destes novos periódicos. Quem não é da área, ou não é conhecedor dos rankings, dificilmente conseguirá avaliar a qualidade.

Em suma, tem havido um aumento do número de periódicos disponíveis nas diversas áreas da ciência, ainda que a muitos falte legitimidade. Para os pesquisadores, a escolha do periódico onde publicar pode ter se tornado substancialmente mais complexa. Como distinguir quais são os bons periódicos e quais são os menos bons? Quais os periódicos que cumprem o processo de revisão editorial? Qual a atenção institucional que é dada ao periódico? Qual o futuro do periódico? Qual o impacto para a carreira, ou simplesmente para a visibilidade do artigo, se for publicado em um ou outro periódico? Estas questões merecem muito mais debate pela comunidade.

\section{Alguns Rankings}

Nossos resultados empíricos implicam que a probabilidade de adoção de uma lista está positivamente correlacionada com o tamanho do departamento e está inversamente correlacionada com a qualidade percebida do departamento. (... ) Há variabilidade considerável entre tais listas e entre diferentes instituiçôes nas percepções da qualidade dos periódicos. Isto sugere que, embora as listas possam reduzir o nivel de incerteza quanto à avaliação da qualidade da pesquisa, fornecendo metas explícitas, as listas também podem induzir os pesquisadores a desenvolver um capital bumano especifico a instituição. Isto poderia reduzir a mobilidade dos pesquisadores e impedir o desenvolvimento da carreira

Van Fleet, McWilliams \& Siegel (2000, p. 839, tradução minha)

Embora todos os acadêmicos usem rankings, mesmo que com diferentes intensidades, poucos conhecem efetivamente o que sustenta os rankings e como se 
processa a classificação dos periódicos nos diversos estratos. Como as realidades são muito distintas é impossível fazer aqui uma análise exaustiva. Há muitos rankings disponíveis, seguindo diferentes metodologias, que classificam os periódicos em Administração. No Brasil, a CAPES usa um sistema, o QUALIS, que é baseado num conjunto de indicadores formais de organização e de processo editorial, de indexação em bases de dados, e de fatores de impacto. Nos EUA é usual cada universidade ter o seu próprio ranking interno (SHUGAN, 2003). Estes rankings internos podem variar substancialmente, mas nas universidades de topo, europeias e norte americanas, é interessantemente bastante homogêneo a acordar num conjunto similar de periódicos. Na Europa, as práticas variam muito e há desde rankings internos (ver a Journal quality list de Harzing), até a adoção dos padrões norte-americanos e até mesmo a ausência de qualquer avaliação de artigos e periódicos. Ainda assim, é expressivo o número de universidades que usam métricas baseadas em fatores de impacto - e esta é uma função quase direta das citações aos artigos publicados no periódico. Por fim, temos universidades que usam apenas a indicação se o periódico é classificado na ISI ou na Scopus.

O índice mais utilizado, ou, pelo menos, o que ainda recebe maior reputação é o ISI Journal Citation Report, que disponibiliza o indicador "fator de impacto" (FI). Em essência o FI indica o número de citações que um periódico recebeu (somando as citações aos artigos que publicou) num período definido - por exemplo, o último ano, os últimos dois anos ou os últimos cinco anos. Mas, importa entender que os fatores de impacto não são estáveis e efetivamente há grandes variações. Por exemplo, na Tabela 1 incluo apenas uma amostra dos 15 principais periódicos classificados na ISI WoK, na categoria de Management, com maiores fatores de impacto em dois períodos: 2000 e 2013. Não apenas a importância relativa dos periódicos varia substancialmente, como, também, notamos que apenas 6 periódicos se mantém no topo da lista em 2013 comparativamente a 2000. Mas, o que realmente significam estas variações? Como se explica que alguns periódicos ascendam rapidamente ao topo dos rankings de citações e outros saiam do ranking? Se tomarmos os rankings como indicadores de qualidade percebida, então as percepções de qualidade variam ao longo do tempo. Importa neste sentido notar que os periódicos mais generalistas (como, por exemplo, os da Academy) parecem ser mais estáveis que os especializados em certas disciplinas (DAVIS, 2014).
Tabela 1. Fatores de impacto em dois momentos: 2000 e 2013

\begin{tabular}{|c|c|c|c|}
\hline Periódico & FI (2000) & Periódico & FI (2013) \\
\hline $\begin{array}{l}\text { Academy of } \\
\text { Mgmt Review }\end{array}$ & 3,912 & $\begin{array}{l}\text { Academy of } \\
\text { Mgmt Review }\end{array}$ & 7,817 \\
\hline $\begin{array}{l}\text { Administrative } \\
\text { Sc. Quarterly }\end{array}$ & 3,333 & $\begin{array}{l}\text { Acad. Mgmt } \\
\text { Annals }\end{array}$ & 7,333 \\
\hline $\begin{array}{l}\text { California } \\
\text { Mgmt Review }\end{array}$ & 2,877 & $\begin{array}{l}\text { Journal of } \\
\text { Management }\end{array}$ & 6,862 \\
\hline $\begin{array}{l}\text { Harvard Bus. } \\
\text { Review }\end{array}$ & 2,561 & MIS Quarterly & 5,405 \\
\hline $\begin{array}{l}\text { Strategic Mgmt } \\
\text { Journal }\end{array}$ & 2,531 & $\begin{array}{l}\text { Academy of } \\
\text { Mgmt Journal }\end{array}$ & 4,974 \\
\hline $\begin{array}{l}\text { Academy of } \\
\text { Mgmt Journal }\end{array}$ & 2,375 & Pers. Psychology & 4,540 \\
\hline MIS Quarterly & 2,064 & $\begin{array}{l}\text { Jrl Operations } \\
\text { Management }\end{array}$ & 4,478 \\
\hline $\begin{array}{l}\text { Sloan Mgmt } \\
\text { Review }\end{array}$ & 1,794 & $\begin{array}{l}\text { Jrl Applied } \\
\text { Psychology }\end{array}$ & 4,367 \\
\hline $\begin{array}{l}\text { Human } \\
\text { Resource Mgmt }\end{array}$ & 1,268 & $\begin{array}{l}\text { Organization } \\
\text { Science }\end{array}$ & 3,807 \\
\hline $\begin{array}{l}\text { Journal of } \\
\text { Management }\end{array}$ & 1,235 & $\begin{array}{l}\text { Jrl Inf. } \\
\text { Technology }\end{array}$ & 3,789 \\
\hline $\begin{array}{l}\text { Organ. Bebav. } \\
\text { Human Dec. }\end{array}$ & 1,200 & $\begin{array}{l}\text { Jrl Supply } \\
\text { Chain Mgmt }\end{array}$ & 3,717 \\
\hline $\begin{array}{l}\text { Management } \\
\text { Learning }\end{array}$ & 1,186 & $\begin{array}{l}\text { Jrl. International } \\
\text { Bus. Studies }\end{array}$ & 3,594 \\
\hline Research Policy & 1,078 & $\begin{array}{l}\text { Org. research } \\
\text { Methods }\end{array}$ & 3,525 \\
\hline $\begin{array}{l}\text { Organization } \\
\text { Science }\end{array}$ & 1,052 & $\begin{array}{l}\text { Jrl Management } \\
\text { Studies }\end{array}$ & 3,277 \\
\hline $\begin{array}{l}\text { Jrl. International } \\
\text { Bus. Studies }\end{array}$ & 1,012 & $\begin{array}{l}\text { Mgmt Organ. } \\
\text { Review }\end{array}$ & 3,277 \\
\hline
\end{tabular}

Fonte: dados de 2000 de JCR Year and Edition: 2000 Social Science e dados de 2013 de JCR Year and Edition: 2013 Social Science (categoria: Management).

Também importa entender que estas listas não têm uma composição estável. Por exemplo, a ISI WoK incluía, apenas e na categoria de Management, 60 periódicos em 2000, mas em 2013 já tinha listados 172 periódicos. Também é relevante entender que há grandes diferenças entre disciplinas. Por exemplo, apesar do grande aumento do fator de impacto de alguns periódicos de Administração, ainda estão longe dos fatores de impacto dos periódicos e outras disciplinas - a revista Nature tem um fator de impacto de 42, a Annual Review of Immunology de 47, a Science de 30, a Physiology Review de 28, a Nature Biotechnology de 39, para citar apenas alguns. 
Outras listas, como a Financial Times 45 (FT45) identificam os principais periódicos do mundo para classificar as escolas de negócios (business schools) de acordo com o seu histórico de publicações científicas nestes periódicos. A classificação FT45 (Tabela 2) não classifica os periódicos, mas distingue os 45 periódicos que utiliza na construção dos rankings das escolas.

Tabela 2. Financial Times 45 (2012)

1. Academy of Management Journal (Academy of Management)

2. Academy of Management Perspectives (Academy of

Management)

3. Academy of Management Review (Academy of Management)

4. Accounting, Organisations and Society (Elsevier)

5. The Accounting Review (American Accounting Association)

6. Administrative Science Quarterly (Cornell University)

7. American Economic Review (American Economic Association)

8. California Management Review (UC Berkeley)

9. Contemporary Accounting Research (Wiley)

10. Econometrica (Econometric Society, Wiley)

11. Entrepreneurship Theory and Practice (Baylor University, Wiley)

12. Harvard Business Review (Harvard Business School Publishing)

13. Human Resource Management (Wiley)

14. Information Systems Research (Informs)

15. Journal of Accounting and Economics (Elsevier)

16. Journal of Accounting Research (University of Chicago, Wiley)

17. Journal of Applied Psychology (American Psychological Association)

18. Journal of Business Ethics (Kluwer Academic)

19. Journal of Business Venturing (Elsevier)

20. Journal of Consumer Psychology (Elsevier)

21. Journal of Consumer Research (University of Chicago)

22. Journal of Finance (Wiley)

23. Journal of Financial and Quantitative Analysis (Cambridge

University Press)

24. Journal of Financial Economics (Elsevier)

25. Journal of International Business Studies (Academy of International Business)

26. Journal of Management Studies (Wiley)

27 Journal of Marketing (American Marketing Association)

28. Journal of Marketing Research (American Marketing Association)

29. Journal of Operations Management (Elsevier)

30. Journal of Political Economy (University of Chicago)

31. Journal of the American Statistical Association (American Statistical Association)

32. Management Science (Informs)

33. Marketing Science (Informs)

34. MIS Quarterly (MIS Research Centre, University of Minnesota)

35. Operations Research (Informs)

36. Organization Science (Informs)

37. Organization Studies (SAGE)

38. Organizational Behaviour and Human Decision Processes (Academic Press)
39. Production and Operations Management (Wiley)

40. Quarterly Journal of Economics (MIT)

41. Rand Journal of Economics (The Rand Corporation, Wiley)

42. Review of Accounting Studies (Springer)

43. Review of Financial Studies (Oxford University Press)

44. Sloan Management Review (MIT)

45. Strategic Management Journal (Wiley)

Nota: os periódicos estão ordenados alfabeticamente. Entre parênteses a editora.

Fonte: http://www.ft.com/cms/s/2/3405a512-5cbb-11e1-8f1f00144feabdc0.html\#axzz3FVEhBBa1

Em muitos países, as escolas (faculdades ou universidades) criam os seus próprios rankings (ver SHARPLIN; MABRY, 1985; EXTEJT; SMITH, 1990; DURDEN; ELLIS, 1994; HOTARD ET AL., 1996). Embora existam inúmeros rankings (recomendo consultar a listagem de Harzing, 2014) o leitor pode consultar os exemplos da HEC Paris, da Cranfield University School of Management e da ESSEC Business School. Usualmente estes rankings servem apenas para avaliações internas dos pesquisadores para decisões de contratação e progressão na carreira. No entanto, estas listas não são inócuas porque em algumas universidades os pesquisadores recebem um prêmio financeiro por artigo publicado em periódicos que constem destas listas. Também a sua progressão na carreira, e a tenure, dependem de as suas publicações serem nestes periódicos. No essencial, o que estas listas pretendem é regular a atividade do pesquisador, reduzindo a incerteza ao oferecer uma pretensa métrica para avaliar a qualidade, mas, sobretudo, oferecer um instrumento que os pesquisadores podem analisar para identificar quais os periódicos em que devem publicar (ROND; MILLER, 2005). Ainda que seja altamente criticável, inclusive pelo jogo de relações de poder que existem dentro dos departamentos que pode levar à inclusão de alguns periódicos e não de outros (ROND; MILLER, 2005), é transparente. Ann-Will Harzing na "Journal quality list" reúne diferentes rankings existentes, muitos de departamentos e universidades, e organizaos de modo que todos possam conhecer, analisar e comparar. Esta lista está disponível em http://www. harzing.com/jql.htm e é atualizada regularmente. Em fevereiro de 2014 lançou a 52 edição, onde inclui 22 rankings com cerca de 1.000 periódicos.

Outro instrumento é o Google Scholar, que se tem se tornado mais relevante e uma alternativa aos indicadores mais tradicionais como a Web of Knowledge. Pelo menos em parte porque o Google é gratuito e de fácil acesso e porque inclui todos os tipos de trabalhos, não apenas artigos publicados em alguns periódicos. 
Uma análise das vantagens e desvantagens do Google Scholar pode ser vista em Jacsó (2005).

No Brasil o Qualis é um caso interessante que merece destaque, em especial por estar em continua adaptação e incorpora um leque alargado de periódicos, num sistema que define estratos e é balizado por quotas.

\section{QUALIS brasileiro}

O QUALIS, no Brasil, é uma iniciativa interessante que veio procurar organizar e promover a qualidade nos periódicos. A "regulação" teve efeito sobretudo em matéria de organização e processos nos periódicos. Ou seja, sobre a legitimação institucional (MEYER; ROWAN, 1977), obrigando a que, no mínimo adotem alguns elementos (ver Tabela 3). Assim, o Qualis não pretende realmente avaliar a qualidade per se dos artigos ou dos periódicos nacionais.

Os critérios de avaliação para o triênio 2010-2012 (Tabela 3) indicam os requisitos mínimos para um periódico ser classificado num dos estratos existentes. Os estratos são A1, A2, B1, B2, B3, B4, B5 e C. Vale salientar, por exemplo, que para ser classificado como B5 é suficiente ter um ISSN e uma periodicidade definida. Para ser classificado como B4 além dos dois aspectos anteriores, deve ter revisão pelos pares, edições atualizadas e indicar as normas de submissão.

Como novos periódicos são adicionados ao Qualis? A inclusão de novos periódicos avaliados em cada área científica ocorre quando um pesquisador afiliado num programa de stricto sensu publica no periódico. Por exemplo, se um pesquisador da área de Administração publicar num periódico de Biotecnologia este periódico será avaliado para posterior classificação e inclusão no Qualis, na área de Administração.

Ao examinar a tabela 3 é interessante notar que, no sistema Qualis, os periódicos podem ascender até ao nível B3 apenas com a organização e transparência do processo editorial e de organização do periódico (ver a respeito o trabalho de Meyer e Rowan, 1977). Para o nível B2 é necessário um fator de maturidade (de apenas 3 anos) e para o nível B1 ser editado por umas das editoras enunciadas e ter cinco anos de existência. A partir deste plateau requer-se a existência de um fator de impacto (H-scopus ou JCR). Também fica evidente que até ao estrato B2 os critérios são fortemente baseados em processo e organização, só a partir daqui exigindo indexadores e, depois, mensuração de fatores de impacto. Assim, em 2014 apenas seis periódicos em Administração são classificados como A2 (RAC,
BAR, RAUSP, RAE, Organizações e Sociedade, BBR) e apenas dois têm fator de impacto ISI (a RBGN, que não é A2, e a RAE). Portanto, todos os A1 são estrangeiros. Mas, mesmo alguns A2 nacionais parecem não cumprir todos os requisitos definidos na própria tabela de classificação.

Tabela 3. Critérios Qualis 2010-2012

\begin{tabular}{|c|c|}
\hline Estrato & Critérios \\
\hline $\mathrm{C}$ & $\begin{array}{l}\text { Periódicos que não atendem aos critérios } \\
\text { para ser B5. }\end{array}$ \\
\hline B5 & $\begin{array}{l}\text { - Ter ISSN; } \\
\text { - Ter periodicidade definida. }\end{array}$ \\
\hline B4 & $\begin{array}{l}\text { Atender aos critérios para se enquadrar no } \\
\text { estrato B5 } \\
\text { - Ter revisão por pares; } \\
\text { - Edições atualizadas; } \\
\text { - Normas de submissão. }\end{array}$ \\
\hline B3 & $\begin{array}{l}\text { Atender aos critérios para se enquadrar } \\
\text { no estrato B4 e atender a } 6 \text { dos critérios } \\
\text { abaixo. } \\
\text { - Missão/foco; } \\
\text { - Informa o nome e afiliação do editor; } \\
\text { - Informa nome e afiliação dos membros } \\
\text { do comitê editorial; } \\
\text { - Divulga anualmente a nominata dos } \\
\text { revisores; } \\
\text { - Mínimo de dois números por ano; } \\
\text { - Informa dados completos dos artigos; } \\
\text { - Endereço de pelo menos um dos autores. }\end{array}$ \\
\hline B2 & $\begin{array}{l}\text { Atender aos critérios para se enquadrar no } \\
\text { estrato B3 e } \\
\text { - Informar sobre os trâmites de aprovação; } \\
\text { - Apresentar a legenda bibliográfica da } \\
\text { revista em cada artigo; } \\
\text { - Ter conselho diversificado; } \\
\text { - Editor chefe não é autor } \\
\text { - Informação sobre processo de avaliação; } \\
\text { - Ter mais de três anos; e } \\
\text { - Ter pelo menos } 1 \text { Indexador (SCOPUS, } \\
\text { EBSCO, DOAJ, GALE, CLASE, HAPI, } \\
\text { ICAP, IBSS) }\end{array}$ \\
\hline B1 & $\begin{array}{l}\text { Atender aos critérios do estrato B2, e } \\
\text { - ter mais de } 5 \text { anos } \\
\text { - } 0<\mathrm{H}-\text { Scopus } \leq 4 \text { ou } 0 \leq \mathrm{JCR} \leq 0,2 \text {, o } \\
\text { que for mais favorável ao periódico. } \\
\text { ou } \\
\text { - Estar na Scielo ou Redalyc } \\
\text { ou } \\
\text { - Ser periódico de uma das seguintes } \\
\text { Editoras: Sage, Elsevier, Emerald, } \\
\text { Springer, Inderscience, Pergamo, Wiley ou } \\
\text { Routledge. }\end{array}$ \\
\hline A2 & $\begin{array}{l}4<\mathrm{H}-\mathrm{Scopus} \leq 20 \text { ou } 0,2<\mathrm{JCR} \leq 1,0, \text { o } \\
\text { que for mais favorável ao periódico. }\end{array}$ \\
\hline
\end{tabular}


A1 Índice $\mathrm{H}$ da Base Scopus (H-Scopus) > 20 ou fator de impacto no Journal of Citation Reports (JCR) $>1,0$, o que for mais favorável ao periódico.

$\mathrm{O}$ índice $\mathrm{H}$ expressa o número de artigos $(\mathrm{H})$ de um periódico que receberam $\mathrm{H}$ ou mais citações nos artigos de uma base definida de periódicos e no caso aqui escolhida a base Scopus. $\mathrm{O}$ índice tenta quantificar o impacto científico do periódico.

O fator de impacto de um periódico divulgado pelo JCR é baseado em dois elementos: o numerador, que é o número de citações no ano corrente a quaisquer itens publicados em um periódico nos últimos n anos, e o denominador, que é o número de artigos publicados nos mesmos $\mathrm{n}$ anos. $\mathrm{O}$ fator de impacto publicado pelo JCR considera as bases da ISI Web of Science da Thomson Scientific Reuters para o cálculo.

Fonte: http://qualis.capes.gov.br/webqualis/publico/ documentosDeArea.seam? conversationPropagation=begin (documento em PDF) acedido 01/10/2014.

O sistema de quotas definido pela Capes estabelece o seguinte: "o número de periódicos classificados como A1 ser menor que o número de periódicos classificados como A2; A1+A2 representar no máximo $25 \%$ do total de periódicos qualificados da área; e a soma dos periódicos em A1, A2 e B1 não ultrapassar $50 \%$ do total de periódicos qualificados pela área" (Documento da área 2013, acesso em http://www.avaliacaotrienal2013.capes.gov.br/ documento-de-area-e-comissao). Assim, também será interessante entender como o Qualis irá evoluir por dois motivos: primeiro, dado que impõe quotas ao percentual de periódicos em cada um dos estratos que define; segundo, porque o Qualis é um sistema que visa à padronização, e melhorar o padrão dos periódicos nacionais. Ou seja, é um sistema que visa legitimar os periódicos, dando-lhes aparência de periódicos científicos, mas não é efetivamente um sistema que garante a qualidade do conteúdo publicado (TRZESNIAK ET AL., 2012).

\section{Problemas com os rankings}

Os métodos para estabelecer rankings podem ser baseados em medidas subjetivas de qualidade por pesquisadores da comunidade (OLTHETEN ET AL., 2005; CURRIE; PANDHER, 2011), mas é mais comum recorrer a medidas objetivas (BAUMGARTNER;
PIETERS, 2003; GEARY ET AL., 2004). As medidas subjetivas são calcadas no julgamento dos pares especialistas. As medidas objetivas têm ganho importância, baseadas na contagem de citações (LEYDESDORFF, 2008; CURRIE; PANDHER, 2011) para aferir fatores de impacto da ISI WoK (ISI Web of Knowledge), Scopus ou outros. Outra medida é a contagem simples do número de artigos publicados (por pesquisadores e universidades) num conjunto de periódicos de topo. Então, o método comum para avaliar qualidade dos artigos é a contagem do número de citações que o periódico (ou o artigo) recebe (SMITH, 2004), assumindo-se que uma citação é uma evidência da relevância para a literatura (PODSAKOFF ET AL., 2005). No entanto, é possível argumentar que o número de citações de um artigo pode identificar impacto, mas não realmente qualidade (SMITH, 2004; LEYDESDORFF, 2008). Por outro lado, somando as citações de todos os artigos publicados num dado periódico podemos calcular a "qualidade" do periódico, mas é argumentável se efetivamente é um bom sistema para avaliar a qualidade individual dos artigos.

Os rankings podem ser especialmente danosos para pesquisadores em disciplinas onde há poucos periódicos com impacto. Por exemplo, no Brasil, há poucos periódicos especializados em Marketing, pelo que os pesquisadores nesta disciplina competem com outros por espaço nos periódicos generalistas de Administração. De igual modo podem ficar em desvantagem relativa com pesquisadores em áreas muito especializadas porque os periódicos destas áreas tendem a ser menos citados (a audiência é menor) e $\operatorname{logo}$ a ter menor fator de impacto, não entrando nas listas de "periódicos de topo". A forma como contemplar estes casos não é evidente e necessita ser debatida.

Ainda uma disfunção prende-se com o idioma. Todos os periódicos de topo apenas publicam artigos em inglês e há grandes disparidades na proficiência no idioma inglês (MAN ET AL., 2004). Conseguir desenvolver a mestria na língua que não prejudique a publicação internacional é extremamente difícil, colocando em desvantagem os pesquisadores de países onde a língua oficial não é o inglês.

Há outros problemas quando se definem listas curtas de periódicos de topo. O Qualis brasileiro não é imune a estas disfunções. Por exemplo, apenas seis periódicos nacionais são classificados como A2 em Administração - BAR, BBR, O\&S, RAE, RAC e 
RAUSP - e mesmo entre estes, um deles só aceita artigos em inglês. Há o risco eventual de os periódicos de topo privilegiarem certos grupos ao forçar a adoção de teorias ou metodologias, excluindo as restantes (UNCLES, 2004). Assim, importa entender que num sistema baseado no histórico de publicações, os periódicos de topo da área podem realmente ter um impacto grande. É, por isso, importante que outros periódicos ascendam nestas listagens. Mas, para esta ascensão não basta seguir procedimentos e que pareçam periódicos de qualidade (MEYER; ROWAN, 1977), é, também, necessário que a comunidade desenvolva a percepção que têm realmente qualidade. O papel do editor é fundamental nesta legitimação.

\section{0 que faz um bom periódico?}

Se debatemos os rankings, interessa, também, entender o que faz um bom periódico. Starbuck (2005) questionou quão melhores são os periódicos de topo e observou que alguns artigos excepcionais foram publicados em periódicos de segunda linha. Davis (2014) questionou porque temos periódicos. Os periódicos são os veículos aceitos para comunicar novos conhecimentos, são fóruns de debate, são instrumentos de preservação do conhecimento, são meios de certificação. Os pesquisadores em Administração buscam os artigos em disciplinas da sua especialidade. Por exemplo, os pesquisadores de Estratégia buscam acompanhar os mais recentes progressos em periódicos como o SMJ, LRP, AMR, AMJ, Org. Sc., JoM entre outros. Ou seja, os periódicos atraem e reúnem comunidades especificas. Os periódicos impõem os artigos recebidos a um sistema editorial, onde editores e revisores servem a função de certificar o conhecimento transmitido. Os periódicos têm ainda a função de sinalizar a qualidade indicando a potenciais leitores o que vale a pena ler. Em especial, a norma assumida é que é preferível ler os artigos publicados em melhores periódicos, do que nos periódicos de nível inferior. Todas estas funções são relevantes para a comunidade acadêmica, especialmente quando consideramos que todos os anos são publicados mais de 8.000 artigos (DAVIS, 2014).

As práticas dos periódicos são bastante diferentes em matéria de publicações. Um número é ilustrativo: o número de artigos que os periódicos publicam anualmente. Num levantamento sumário realizado para este artigo, apenas dos artigos publicados em 2013, o Administrative Science Quarterly publicou 17 artigos, Academy of Management Review 29 artigos e o Academy of Management Journal 74 artigos. Estas são já diferenças substanciais que ainda contrastam com 331 artigos publicados no Journal of Business Researche 341 no Journal of Business Ethics. Podemos usar os números de artigos publicados como indicador de exigência e qualidade das publicações? Pelo menos em parte parece ser esse o argumento de periódicos internacionais de topo como o ASQ (ver DAVIS, 2014).

Os periódicos diferem também nas exigências impostas no processo de revisão. De modo geral, os principais periódicos de Administração têm taxas de rejeição superiores a 90\% (FERREIRA, 2013; SUGIMOTO ET AL., 2013) pressupondo-se que os revisores e editores apenas escolhem para publicação os artigos que são realmente melhores. $\mathrm{O}$ processo de revisão pelos pares é o mecanismo de garantia de qualidade do que é publicado (SHUGAN, 2007; LEWIN, 2014), mas não é um sistema perfeito (ROTH, 2002; FREY, 2003). É este processo de revisão pelos pares, em especial observando o sistema de blind review, que certifica o conhecimento. Assim, um processo editorial eficiente, eficaz e transparente é essencial. Embora não seja tão evidente como revelar a transparência que efetivamente decorre, é interessante notar que um dos requisitos definidos pela CAPES, no Qualis, é que os periódicos revelem anualmente a lista dos revisores que usaram (embora muitos periódicos não incluam esta lista, todos disponibilizam uma lista de corpo de revisores).

Os periódicos também diferem no esforço para aumentar a sua qualidade, conseguir indexações, atrair os melhores trabalhos, garantir a eficácia do sistema de revisão pelos pares selecionando os melhores revisores (SHUGAN, 2007; LEWIN, 2014), etc. Esta é uma das funções dos editores. Como um dos indicadores de qualidade dos periódicos é o fator de impacto e citações aos artigos publicados, infelizmente alguns editores podem ser tentados a usar de expedientes para promover os seus periódicos. Por exemplo, forçando a citação aos artigos publicados no seu periódico, ou buscar conluio com outros editores para promover a citação cruzada (ARNOLD; FOWLER, 2011; VAN NOORDEN, 2013; DAVIS, 2014). Estas práticas aumentam as citações sem alterações de qualidade, desvirtuando o fator de impacto como métrica de qualidade relativa.

Então, o que faz um bom periódico? Em essência, o bom periódico é o que cumpre os requisitos formais (ver, por exemplo, os critérios do Qualis), tem um sistema editorial eficiente, eficaz e que garante qualidade de todos os agentes envolvidos, que procura a sua promoção por meios legítimos e que divulga o 
mais generalizadamente possível os artigos que publica. Mas, os bons periódicos também procuram a melhoria da ciência. Isto significa que os periódicos são, também, instrumentos para a melhoria do trabalho científico. Os artigos que são submetidos a um processo editorial, com revisão pelos pares, devem apresentar melhorias. Isto porque os revisores e editores também têm a função construtiva de auxiliar os autores a aprimorar os seus artigos (LEWIN, 2014). Estes aprimoramentos podem incidir em aspetos de redação e clareza, mas, também, de rigor metodológico, de contribuição, de embasamento teórico fundamental (LEWIN, 2014). Nas ciências sociais não é suficiente ter bons resultados empíricos, é, também, preciso comunicar adequadamente a mensagem.

\section{A revisão pelos pares}

Davis (2014) propôs que o fundamental nos periódicos atuais não está na distribuição, ou disseminação, do conhecimento, mas sim no processo de revisão dos artigos. É o processo de revisão que melhor distingue a qualidade dos periódicos. Em especial, Davis aponta que o processo editorial reflete a proposição do que é uma contribuição e como esta deve ser avaliada. É no processo de revisão que a comunidade certifica as contribuições e decide quais os trabalhos que vale a pena ler.

Esta análise de Davis (2014) está longe de ser neutra por diversos motivos. Primeiro, sabemos que muitos periódicos (inclusive brasileiros) têm dificuldades sérias com as revisões dos seus artigos (não cumprem os prazos, os comentários dos revisores são fracos e pouco construtivos, são permeáveis a amizades, etc.). Segundo, importaria definir quem são os gatekeepers que decidem pela comunidade. Ou seja, quem são os revisores, os editores e editores adjuntos e os membros do corpo editorial dos periódicos? Terceiro, precisamos avaliar o funcionamento do processo editorial. Quarto, necessitamos avaliar a composição dos corpos editoriais e analisar a sua diversidade, inclusive observando se há endogenia.

O processo de revisão pode ser bem demorado. Phelan et al. (2002) mostraram que entre a submissão inicial e a publicação dos artigos podem decorrer alguns anos. Cada nova ronda de revisão pode estender-se por alguns meses. A justificação usual é que o trabalho dos revisores é voluntário e gratuito e dependente da sua boa vontade. Muitas vezes os pareceres dos revisores são fracos e enviesados por preferências pessoais. Mas, um processo editorial mal gerido pode prejudicar seriamente a carreira de jovens acadêmicos que dependem das publicações para a tenure ou para prestar um concurso.

Há outras disfunções do sistema de revisão pelos pares. Por exemplo, é hoje uma norma aceita que um artigo tem de ter uma contribuição. Certamente faz sentido sugerir que há um propósito que levou o pesquisador a fazer o trabalho, escrever o artigo e submeter. Mas, a essência do que é uma contribuição não é tão evidente nem universalmente aceita. A contribuição como é comumente entendida é uma contribuição para a teoria (em periódicos dirigidos para os practitioners - os práticos, gestores ou executivos para a prática). Excluídos de publicação ficarão outros artigos sobre temas que são relevantes, inclusive para a comunidade científica, mas que não sigam um padrão de contribuição como o descrito. Assim, por exemplo, é difícil publicar um ensaio como este onde debato, na primeira pessoa e assumindo a propriedade, na vOz ativa e sem o uso de expressões indefinidas. Este artigo não tem uma contribuição teórica segundo os cânones vigentes. Mas, tem uma contribuição para a comunidade.

\section{Discussão e notas finais}

A comunidade acadêmica concorda, pelo menos na generalidade, que a publicação de artigos em periódicos científicos é o principal meio para a divulgação do conhecimento. Assim, a aferição da qualidade do veículo de comunicação do conhecimento - os periódicos - parece pertinente. É importante para os pesquisadores saberem distinguir os periódicos e que os rankings são instrumentos úteis, ainda que não perfeitos, para rapidamente aferir a qualidade dos periódicos e decidir para onde submeter os artigos.

O sistema brasileiro tem vindo a passar por uma evolução acelerada, mas não sem críticas (ROCHAE-SILVA, 2009). Como sistema móvel, que é ajustado periodicamente, busca usar métricas objetivas que ainda são muito fundeadas em aspectos formais para os estratos mais baixos e em citações, ou fatores de impacto, para os estratos mais altos. A promoção de veículos científicos nacionais é interessante num quadro de apoio e legitimação regulada, e tornam o Qualis um dos sistemas mais avançados. No entanto, é relevante atender que o desenvolvimento de uma cultura nacional de pesquisa demora tempo e que se o privilégio for posto exclusivamente nas publicações em periódicos internacionais, isto pode matar o sistema nacional. Neste caso é provável que o próprio 
sistema se torne paroquial e fechado. Possivelmente, de acordo com a teoria institucional (DIMAGGIO; POWELL, 1983), serão as instituições líder e elitistas a encabeçar as mudanças. Mas, apesar de os progressos nacionais serem assinaláveis, na maioria das disciplinas ainda estamos longe da ciência praticada no hemisfério norte (CABRAL; LAZZARINI, 2011).

No Brasil critica-se o produtivismo acadêmico (FARIA, 2011). Em essência, as críticas fundamentamse em os pesquisadores serem avaliados pela sua produção científica (não de ensino ou outra) e neste sistema tender a conduzir à produção em quantidade em relativo desfavor da qualidade. No entanto, é difícil avaliar até que ponto existe uma pressão para publicar no Brasil. Ou seja, o produtivismo é resultado de pressões institucionais, de motivações individuais dos pesquisadores mais prolíficos, ou dos pares? A Capes classifica como muito bom o pesquisador que publique 150 pontos por triênio (triênio 20122015), o que realmente significa publicar um artigo num periódico do estrato B2 por ano, em média. Este nível de exigência não parece exagerado. Assim, não é evidente que exista uma pressão institucional pelas agências reguladoras. Mas, o produtivismo pode, ainda assim, estar a ser promovido Por exemplo, como o nível de 150 pontos, que a CAPES considera de muito bom, permite construir um currículo científico passível de ver o reconhecimento pela atribuição de uma bolsa de produtividade? É, ainda, necessária muita pesquisa para entendermos realmente qual o estado real e emocional da comunidade científica em Administração no Brasil.

\section{Limitações e pesquisa futura}

Este artigo é um ensaio conceitual sobre uma questão que importa à comunidade pelo impacto que tem sobre a sua atividade, reputação ou conhecimento dos agentes envolvidos e sobre a regulação. Sendo um ensaio conceitual, que visa iniciar um debate que importa ter, não propõe nem testa hipóteses, nem faz uso de dados empíricos. Também não teve o objetivo de comparar rankings, nem propor novos modelos de avaliação. Apenas visa lançar um debate que tem estado silencioso no meio acadêmico formal brasileiro, embora muito debatido em fóruns informais e "entre amigos". Em grande medida os debates informais têm estado centrados apenas no produtivismo acadêmico, mas é necessário alargar o debate aos rankings, aos sistemas de incentivo, às carreiras, à promoção de pesquisa com penetração internacional.
Pesquisa futura também será importante analisando como os pesquisadores da área percepcionam a importância de publicar internacionalmente em periódicos com fator de impacto. Esta questão é especialmente relevante face ao atual sistema de incentivos em que, pelo menos formalmente, o sistema de pontos não distingue a qualidade dos periódicos e os pesquisadores podem realizar os 150 pontos definidos na avaliação da Capes com diferentes combinações de periódicos. Uma das vias para esta pesquisa é coletar dados, possivelmente por meio de questionário, sobre as dificuldades sentidas para conseguir desenvolver pesquisa de alto nível passível de ser aceite em periódicos internacionais de alta reputação. Esta pesquisa pode ser orientadora para a definição de formas de incentivo mais ajustadas aos objetivos da organização (da universidade).

É interessante notar que no Brasil a disponibilização gratuita em acesso livre contribui positivamente para a avaliação dos periódicos. Assim, virtualmente todos os periódicos brasileiros estão disponíveis gratuitamente na internet. No entanto, o sistema brasileiro é dual. Por um lado temos os periódicos associados às associações (como a ANPAD, com a BAR, RAC e TAC), e por outro, a maioria dos periódicos de propriedade de universidades que assumem todos os encargos. Internacionalmente a situação é diferente. Embora alguns periódicos sejam patrocinados por Associações (como os periódicos da Academy of Management ou da Strategic Management Society), a maioria dos periódicos são um negócio em que as editoras internacionais, como a Emerald e Elsevier, investem tempo, recursos humanos e capital. A fonte de receita das editoras está na venda do acesso aos periódicos, que em alguns casos pode custar milhares de reais. Para este negócio, os pesquisadores contribuem com os artigos (e não recebem pagamento por isso), sendo editores (usualmente função não remunerada) e revisores (também sem remuneração).

A multiplicação do número de periódicos, inclusive no Brasil, tem alguns efeitos perniciosos quando os pesquisadores individualmente, e a comunidade no seu conjunto, têm dificuldade de avaliar os periódicos. O problema não está em ter periódicos de acesso livre. Na realidade o acesso livre começou à cerca de uma década com periódicos científicos com revisão pelos pares e reconhecidos pela sua qualidade, como os da Public Library of Science (PLoS) (The New York Times, 2013). O problema pode estar na incapacidade de reconhecer e distinguir qualidades relativas originadas no não respeito pelas normas que a própria academia 
estabeleceu. Ou seja, é relevante debater o fenômeno do open access quando, ao diminuir as barreiras a novas entradas, se reflete no surgimento de novos periódicos que publicam qualquer coisa por um pagamento (The New York Times, 2013) e desvirtuando a filosofia de divulgação livre do conhecimento que presidiu à sua criação.

\section{Considerações finais}

Embora existam inúmeras disfunções no sistema vigente de avaliação da produção científica por meio de medidas quantitativas que têm mais em conta o periódico em que se publica do que a efetiva análise do que foi publicado, este é um sistema em contínuo ajustamento. Os rankings são úteis para os autores, leitores einstituições. Por exemplo, são úteis para ajudar os estudantes de mestrado ou doutorado a identificar quais artigos ler e referenciar. Também são úteis como orientação para os pesquisadores de a quais periódicos submeter os seus artigos. Em especial, atualmente, face à contínua emergência de novos periódicos do tipo "pagar para publicar", são especialmente importantes para distinguir os periódicos. Também para as agências são úteis na medida em que estas precisam ter modos simples, e aceitos, para fazer as avaliações de um grande volume de informação. Ou seja, os rankings, sinalizam qualidade a potenciais interessados. No entanto, importa entender que se há uma associação direta entre a posição do periódico no ranking e a avaliação do currículo do pesquisador, esta relação precisa ser contextualizada. Talvez a avaliação da produção científica do pesquisador necessite de conter aspectos de natureza qualitativa que vá além destes indicadores.

Embora existam muitas disfunções na forma como os rankings são construídos, na adoção de rankings pelas instituições, no foco da publicação pela publicação para satisfazer critérios quantitativos, na existência de publicações de menor qualidade onde o pagamento da "publication fee" parece ser suficiente, a publicação em periódicos ainda tem um papel fundamental na Academia. Os periódicos tradicionais são os veículos aceitos e institucionalizados de divulgação das pesquisas e dos novos conhecimentos gerados. É neste contexto que os rankings de periódicos também ganham algum valor ao juntar audiências, certificar novos conhecimentos, sinalizar o que vale a pena ser lido, orientar para onde vale a pena direcionar os esforços, etc. Os periódicos são indispensáveis ao progresso da ciência e os rankings, pela sua mera existência, são uma força que contribui para aprimoramentos no processo de desenvolvimento científico.

O debate que se propõe é realmente importante porquanto as métricas de avaliação no Brasil estão em construção e evolução. Este processo necessita da participação da comunidade de modo a definirem-se os padrões de qualidade e as normas que serão usadas pelas agências reguladoras. Pesquisa futura poderá ser relevante ao comparar a situação brasileira, no seu atual estado de evolução, com realidades distintas em outros países. Por exemplo, o QUALIS brasileiro tem virtudes, mas ainda parece combinar medidas de organização dos periódicos, medidas objetivas e subjetivas. Qual é a evolução expectável do Qualis e como este se compara às métricas internacionalmente adotadas? Pesquisa futura também será relevante para observar as mudanças nos sistemas de incentivos à produção de maior quantidade ou qualidade. Em muitas universidades brasileiras (mais especialmente as privadas) não há um sistema de carreiras que utilize realmente a produção científica como métrica para decidir a progressão. Assim, qual o impacto dos incentivos, por exemplo, financeiros, nas instituições que já instituíram prêmios por produção? Este debate sobre o papel da pesquisa, dos periódicos e dos rankings previsivelmente contribuirá para reforçar a ciência no Brasil.

\section{Referências}

ARCHAMBAULT, E; LARIVIÈRE, V. History of the journal impact factor: Contingencies and consequences. Scientometrics, v. 79, n. 3, p. 635649, 2009.

ARNOLD, D.; FOWLER, K. Nefarious numbers. Notices of the American Mathematical Society, v.58, p.434-437, 2011.

AZAR, O.; BROCK, D. A citation-based ranking of strategic management journals. Journal of Economics \& Management Strategy, v.17, n.3, p.781-802, 2008.

BADEN-FULLER, C.; RAVAZZOLO, F.; SCHWEIZER, T. Making and measuring reputations: The research ranking of European business schools. Long Range Planning, v.33, n.5, p.621-650, 2000.

BAUMGARTNER, H.; PIETERS, R. The structural influence of Marketing journals: A citation analysis of the discipline and Its subareas over time. Journal 
of Marketing, v.67, p.123-139, 2003.

BERTERO, C.; ALCADIPANI, R.; CABRAL,S.; FARIA, A.; ROSSONI, L. Os desafios da produção de conhecimento em Administração no Brasil. Cadernos EBAPE.BR, v.11, n.1, p.181-196, 2013.

BJÖRK, B.; ROOS, A.; LAURO, M. Global annual volume of peer reviewed scholarly articles and the share available via Open Access options. Proceedings ELPUB 2008 Conference on Electronic Publishing. Toronto, Canada, Junho 2008, p. 1-10.

CABRAL, S.; LAZZARINI, S. Internacionalizar é preciso, produzir por produzir não é preciso. Organizações \& Sociedade, v.18, n.58, p.541-542, 2011.

CAMPOS, J. Qualis periódicos: conceitos e práticas nas Engenharias I. Revista Brasileira de PósGraduação, v. 7, n. 14, p. 477-503, 2011.

CURRIE, R.; PANDHER, G. Finance journal rankings and tiers: An Active Scholar Assessment methodology. Journal of Banking \& Finance, v.35, n.1, p.7-20, 2011.

DAVIS, G. Editorial essay: Why do we still have journals? Administrative Science Quarterly, v.59, n.2, p.193-201, 2014.

DE MEIS, L.; VELLOSO, A.; LANNES, D.; CARMO, M.; DE MEIS, C. The growing competition in Brazilian science: Rites of passage, stress and burnout. Brazilian Journal of Medical and Biological Research, v.36, n.9, p.1135-1141, 2003.

DIMAGGIO, P.; POWELL, W. The iron cage revisited: Institutional isomorphism and collective rationality in organizational fields. American Sociological Review, v.48, p.147-160, 1983.

DINIZ, E. Editorial. RAE Revista de

Administração de Empresas, v. 50 n. 4. p. 353, 2010.

DUBOIS, F.; REEB, D. Ranking the international business journals. Journal of International Business Studies, v.31, n.4, p.689-704, 2000.

DURDEN, G.; ELLIS, L. A method for identifying the most influential articles in an academic discipline. Atlantic Economic Journal, v.21, n.4, p. 1-10, 1994.

EXTEJT, M.; SMITH, J. The behavioral sciences and management: An evaluation of relevant journals. Journal of Management, v.16, n.3, p.539-551, 1990.
FARIA, A. Repensando produtivismo em gestão no (e a partir do) Brasil. Cadernos EBAPE.BR, v.9, n. 4, p.1164-1173, 2011.

FERREIRA, M. O processo editorial: Da submissão à rejeição (ou aceite). Revista Ibero-Americana de Estratégia, v.12, n.3, p.1-11, 2013.

FREY, B. Publishing as prostitution? Choosing between one's own ideas and academic success.

Public Choice, v.116, p.205-223, 2003.

FRIGERI, M.; MONTEIRO, M. Abrindo a caixa preta do Qualis: entendendo a avaliação dos periódicos científicos no Brasil. Anais do Encontro Anual da ANPOCS. 36. 2010.

GARFIELD, E. Citation Indexes for Science. Science, v. 122, n. 1, p. 108-111, 1955.

GARFIELD, E. The history and meaning of the journal impact factor. JAMA, v. 295, n. 1, p. 90-93, 2006.

GEARY, J.; MARRIOTT, L.; ROWLINSON, M. Journal rankings in Business and Management and the 2001 Research Assessment Exercise in the UK. British Journal of Management, v.15, p.95-141, 2004.

GROSS, P.; GROSS, E. College libraries and chemical education. Science, v. 66 n. 1713, p. 385389, (1927).

HOJAT, M.; GONNELLA, J.; CAELLEIGH, A. Impartial judgment by the "gatekeepers" of science: Fallibility and accountability in the peer review process. Advances in Health Sciences Education, v.8, n.1, p.75-96, 2003.

HOTARD, D.; TANNER, J.; MANAKYAN, H. Management faculty ratings of journal quality: The factors and the journals. Southern Business Review, v.22, n.1, p.8-20, 1996.

JACSÓ, P. Google Scholar: The pros and the cons. Online Information Review, v.29, n.2, p.208-214, 2005.

KOLATA, G. Scientific articles accepted (personal checks, too). New York Times, 7 abril 2013. Acesso 07/10/2014 em: http:// www.nytimes.com/2013/04/08/health/forscientists-an-exploding-world-of-pseudo-academia. $\underline{h t m l \text { ?pagewanted }=\text { all \& } \_r=0}$

LARSEN, P.; VON INS, M. The rate of growth in scientific publication and the decline in coverage provided by Science Citation Index. Scientometrics, 
v,84, n.3, p.575-603, 2010.

LEWIN, A. The peer-review process: The good, the bad, the ugly, and the extraordinary. Management and Organization Review, v.10, n.2, p.167-173, 2014.

LEYDESDORFF, L. Caveats for the use of citation indicators in research and journal evaluations.

Journal of the American Society for Information Science and Technology, v.59, p.278-287, 2008.

MAN, J.; WEINKAUF, J.; TSANG, M.; SIN, D. Why do some countries publish more than others? An international comparison of research funding, English proficiency and publication output in highly ranked general medical journals. European Journal of Epidemiology, v.19, p.811-817, 2004.

MARTINS, C.; MACCARI, E.; Silva, A.; Todorov, M. Retrato dos periódicos científicos de secretariado no Brasil. EccoS Revista Científica, n. 34, p. 63-83, 2014.

MEYER, J.; ROWAN, B. Institutionalized organizations: Formal structure as myth and ceremony. American Journal of Sociology, v.83, p.340-363, 1977.

OLTHETEN, E.; THEOHARAKIS, V.; TRAVLOS, N. Faculty perceptions and readership patterns of finance journals: A global view. Journal of Financial and Quantitative Analysis, v.40, n.1, p.223-239, 2005.

PECHLANER, H.; ZEHRER, A.; MATZLER, K.; ABFALTER, D. A ranking of international tourism and hospitality journals. Journal of Travel Research, v.42, n.4, p.328-332, 2004.

PEFFERS, K.; TANG, Y. Identifying and evaluating the universe of outlets for information systems research: Ranking the journals. Journal of Information Technology Theory and Application, v.5, n.1, p.63-84, 2003.

PFEFFER, J.; FONG, C. The business school "business": Some lessons from the US experience.

Journal of Management Studies, v.41, n.8, p.15011520, 2004.

PHELAN, S.; FERREIRA, M.; SALVADOR, R. The first twenty years of the Strategic Management Journal: 1980-1999. Strategic Management Journal, v.23, p.1161-1168, 2002.

PODSAKOFF, P.; MACKENZIE, S.; BACHRACH, D.; PODSAKOFF, $\mathrm{N}$. The influence of management journals in the 1980s and 1990s. Strategic
Management Journal, v.26, n.5, p.473-488, 2005.

CAMPOS, J. Qualis periódicos: conceitos e práticas nas Engenharias I. Revista Brasileira de PósGraduação, vol. 7, n. 14, p. 477-503, 2010.

RITZBERGER, K. A ranking of journals in economics and related fields. German Economic Review, v. 9, n. 4, p. 402-430, 2008.

ROCHA-E-SILVA, M. Comentário editorial. O novo Qualis, ou a tragédia anunciada. Clinics, v.64, n.1, p.1-4, 2009.

ROND, M.; MILLER, A. Publish or perish. Bane or boon of academic life? Journal of Management Inquiry, v.14, n.4, p.321-329, 2005.

ROTH, W-M. Editorial power/authorial suffering. Research in Science Education, v.32, p.215-224, 2002.

SAMKIN, G. Academic publishing: A Faustian bargain? Australasian Accounting Business and Finance Journal, v.5, n.1, p.19-34, 2011.

SERRA, F.; FIATES, G.; FERREIRA, M. Publicar é difícil ou faltam competências? O desafio de pesquisar e publicar em revistas científicas na visão de editores e revisores internacionais. Revista de Administração Mackenzie, v.9, p.32-55, 2008.

SHARPLIN, A.; MABRY, R. The relative importance of journals used in management research: An alternative ranking. Human Relations, v.38, p.139149, 1985.

SHUGAN, S. Journal rankings: Save the outlets for your research. Marketing Science, v.22, n.4, p.437441, 2003.

SHUGAN, S. The editor's secrets. Marketing Science, v.26, n.5, p.589-595, 2007.

SMITH, S. Is an article in a top journal a top article. Financial Management, v.33, p.133-149, 2004.

STAHL, M.; LEAP, T.; WEI, Z. Publications in leading management journals as a measure of institutional research productivity. Academy of Management Journal, v.31, n.3, p.707-720, 1988.

STARBUCK, W. How much better are the mostprestigious journals? The statistics of academic publication. Organization Science, v.16, n.2, p.180200, 2005.

STEWARD, M.; LEWIS, B. A comprehensive analysis of marketing journal rankings. Journal of Marketing Education, v.32, n.1, p.75-92, 2010.

SUGIMOTO, C.; LARIVIÈREB, V.; NIA, C.; 
CRONIN, B. Journal acceptance rates: A crossdisciplinary analysis of variability and relationships with journal measures. Journal of Informetrics, v.7, p.897-906, 2013.

TIMOTHY, C.; WRIGHT, M. Reviewing journal rankings and revisiting peer reviews: Editorial perspectives. Journal of Management Studies, v.44, n.4, p.612-621, 2007.

TREVINO, L.; MIXON, F.; FUNK, C.; INKPEN, A. A perspective on the state of the field: International business publications in the elite journals as a measure of institutional and faculty productivity. International Business Review, v.19, p.378-387, 2010.

TRZESNIAK, P.; PLATA-CAVIEDES, T.;

CÓRDOBA-SALGADO, O. Qualidade de conteúdo, o grande desafio para os editores científicos. Revista Colombiana de Psicologia, v.21, n.1, p.57-78, 2012.

UNCLES, M. Journal rankings: How much credence should we give them? Australasian Marketing Journal, v.12, n.2, p. p.67-72, 2004.

VAN FLEET, D.; MCWILLIAMS, A.; SIEGEL, D. A theoretical and empirical analysis of journal rankings: The case of formal lists. Journal of Management, v.26, n.5, p.839-861, 2000.

VAN NOORDEN, R. Brazilian citation scheme outed. Nature, v.500, p.510-511, 2013.

WARE, M.; MABE, M. The stm report: An overview of scientific and scholarly journal publishing. The Hague, Netherlands: International Association of Scientific, Technical and Medical Publishers, 2012. Acesso dia 07/10/2014 em http://www.stmassoc.org/2012_12_11_STM_Report_2012.pdf 